\title{
Reachable Set Bounding for a Class of Nonlinear Time-Varying Systems with Delay
}

\author{
Xingao Zhu, ${ }^{1}$ Yuangong Sun $\mathbb{D}^{1},{ }^{1}$ and Fanwei Meng ${ }^{2}$ \\ ${ }^{1}$ School of Mathematical Sciences, University of Jinan, Jinan, Shandong 250022, China \\ ${ }^{2}$ School of Mathematical Sciences, Qufu Normal University, Qufu, Shandong 273165, China \\ Correspondence should be addressed to Yuangong Sun; sunyuangong@163.com
}

Received 3 January 2018; Revised 28 February 2018; Accepted 8 March 2018; Published 11 April 2018

Academic Editor: Xin Yu

Copyright (c) 2018 Xingao Zhu et al. This is an open access article distributed under the Creative Commons Attribution License, which permits unrestricted use, distribution, and reproduction in any medium, provided the original work is properly cited.

\begin{abstract}
We investigate the problem of reachable set bounding for a class of continuous-time and discrete-time nonlinear time-varying systems with time-varying delay. Unlike some preceding works, the involved disturbance input and time-varying delay are not assumed to be bounded. By employing an approach which does not involve the conventional Lyapunov-Krasovskii functional, new conditions are proposed such that all the state trajectories of the system converge asymptotically within a ball. Two illustrative examples are also given to show the effectiveness of the obtained results.
\end{abstract}

\section{Introduction}

Reachable set bounding plays an important role in ensuring safe operation in practical engineering through synthesizing controllers to avoid undesirable (or unsafe) regions in the state space [1]. Therefore, the problem of reachable set bounding with different dynamics has been investigated by many researchers in recent years in [2-7], to name a few.

Time delay has received a lot of attention due to its common presence in practical engineering and its detrimental effects on stability [8-14] and performance of systems such as oscillation [15-19]. Therefore, the problem of the reachable set bounding for systems with delay becomes very important. By using the Lyapunov-Razumikhin approach, an ellipsoid was given to bound the reachable set of linear systems with delay and bounded peak inputs in [20]. An improved condition for reachable set bounding for linear systems with delay was proposed in [21] by virtue of a Lyapunov-Krasovskii type functional and the rate of delay. Less conservative estimation results on the reachable set for delayed systems with polytopic uncertainties were established in $[22,23]$ by choosing pointwise maximum Lyapunov functional corresponding to a vertex of the polytope. A delay-partitioning method was applied to study the reachable set bounding problem of delayed systems in [24], which further reduced the conservatism of some existing results. By using the Lyapunov method, LMI conditions for the existence of ellipsoid-based bounds of reachable sets of a linear uncertain discrete system were given in [25].

However, most of the aforementioned works on the reachable set bounding have been mainly focused on linear time-delay systems with constant matrices or a combination of constant matrices (polytopic uncertainties). It seems to us that little has been known about the explicit estimation of reachable set for nonlinear time-varying systems with timevarying delay. Note that it is difficult to apply the usual Lyapunov-Krasovskii functional method to time-varying systems, because it may lead to unsolvable matrix Riccati differential equations or indefinite linear matrix inequalities.

Based on a method developed in positive systems which does not involve the Lyapunov-Krasovskii functional, a delayindependent condition was derived such that all the state trajectories of linear time-varying systems converge exponentially within a ball in [26]. Recently, the result in [26] was extended to homogeneous positive systems of degree one with time-varying delays in [27]. For a class of nonlinear time-delay systems with bounded disturbances, a new approach to obtain the smallest box which bounds all reachable sets was proposed in [28]. For a switched system with nonlinear disturbance which can be bounded by a linear system, global exponential stability criteria were established in [29]. 
Inspired by this and motivated by the work in $[30,31]$, the paper will introduce a new approach which is different from the Lyapunov-Krasovskii functional method to derive new explicit conditions such that all the state trajectories of a class of continuous-time and discrete-time nonlinear time-varying systems with delay converge asymptotically within a ball. The main contribution of this paper is threefold: (1) the nonlinear term considered in this paper takes the more general form, which contains the systems studied in [26-29] as special cases; (2) the involved disturbance input and time-varying delay are not assumed to be bounded; (3) unlike some existing works, we do not need to transform the system to a timeinvariant one, which leads to less conservative conditions for reachable set bounding.

The rest of this paper is briefly outlined as follows. In Section 2, we present the notation used through this paper as well as preliminaries for our results. Section 3 then focuses on deriving explicit conditions under which all the state trajectories of the system converge asymptotically within a ball. Section 4 provides two illustrative examples to show the effectiveness of the obtained results. The paper is concluded in Section 5.

\section{Preliminaries}

Throughout this paper, the following notation will be used. Let $\mathbb{R}^{n}$ and $\mathbb{R}^{n \times n}$ denote the set of $n$-dimensional real vectors and the $n$-dimensional real Euclidean space, respectively. Denote $\mathbb{N}_{0}=\{0,1,2, \ldots\}$ and $\langle n\rangle=\{1,2, \ldots, n\}$. The matrix $A \in \mathbb{R}^{n \times n}$ is said to be Metzler if all its off-diagonal entries are nonnegative. For $x \in \mathbb{R}^{n}$, we denote by $x_{i}$ the $i$ th coordinate of $x$. For two vectors $x, y \in \mathbb{R}^{n}$, we write $x>y$ if $x_{i}>y_{i}$, $x \geq y$ if $x_{i} \geq y_{i}, x \prec y$ if $x_{\mathrm{i}}<y_{i}, x \preceq y$ if $x_{i} \leq y_{i}$, $i \in\langle n\rangle$. Let $\mathbb{R}_{+}^{n}=\left\{x \in \mathbb{R}^{n}: x \geq 0\right\}$. Given $x \in \mathbb{R}^{n}$, set $\|x\|_{\infty}=\max _{i \in\langle n\rangle}\left|x_{i}\right|$ and $|x| \in \mathbb{R}_{+}^{n}$ whose $i$ th coordinate is $\left|x_{i}\right|$. The weighted $\infty$-norm of the vector $x \in \mathbb{R}^{n}$ is defined by $\|x\|_{\infty}^{v}=\max _{i \in\langle n\rangle}\left(\left|x_{i}\right| / v_{i}\right)$, where $v>0$ is an $n$-dimensional vector.

We first consider the continuous-time nonlinear timevarying system with delay

$$
\begin{aligned}
& \dot{x}(t)=F(t, x(t))+G(t, x(t-\tau(t)))+w(t), \quad t \geq 0, \\
& x(t)=\phi(t), \quad t \in\left[-\tau_{\max }, 0\right]
\end{aligned}
$$

where $x(t) \in \mathbb{R}^{n}$ is the state vector, the time-varying delay $\tau(t)$ is continuous on $[0, \infty)$ and satisfies $t-\tau(t) \geq-\tau_{\max }$ with $\tau_{\max }>0, w(t):[0, \infty) \rightarrow \mathbb{R}^{n}$ is the continuous disturbance input, $\phi \in \mathscr{C}\left(\left[-\tau_{\max }, 0\right], \mathbb{R}^{n}\right)$ is the continuous vector valued function specifying the initial state of the system, and the vector fields $F(t, x), G(t, x):[0, \infty) \times \mathbb{R}^{n} \rightarrow \mathbb{R}^{n}$ are continuous and locally Lipschitz with respect to $x$, which ensures the existence and uniqueness of solutions of system (1) [32].

We also consider the following discrete-time nonlinear time-varying system with delay described by

$$
\begin{array}{r}
x(k+1)=F(k, x(k))+G(k, x(k-d(k)))+w(k), \\
k \in \mathbb{N}_{0},
\end{array}
$$

$$
x(k)=\phi(k), \quad k \in\left\{-d_{\max }, \ldots, 0\right\},
$$

where $x(k) \in \mathbb{R}^{n}$ is the state vector, the time-varying delay $d(k): \mathbb{N}_{0} \rightarrow \mathbb{N}_{0}$ satisfies $k-d(k) \geq-d_{\max }$ with $d_{\max }>0$, the vector fields $F, G: \mathbb{N}_{0} \times \mathbb{R}^{n} \rightarrow \mathbb{R}^{n}, w(k): \mathbb{N}_{0} \rightarrow \mathbb{R}^{n}$ is the disturbance input, and $\phi:\left\{-d_{\max }, \ldots, 0\right\} \rightarrow \mathbb{R}^{n}$ is the vector sequence specifying the initial state of the system.

We first extend some definitions given in [33] to the time variant case.

Definition 1. A continuous vector field $f(t, x):[0, \infty) \times$ $\mathbb{R}^{n} \rightarrow \mathbb{R}^{n}$, which is continuously differentiable with respect to $x$ on $\mathbb{R}^{n} \backslash\{0\}$, is said to be cooperative if the Jacobian matrix $(\partial f / \partial x)(t, a)$ is Metzler for all $t \in[0, \infty)$ and $a \in \mathbb{R}_{+}^{n} \backslash\{0\}$.

Definition 2. $g:[0, \infty) \times \mathbb{R}^{n} \rightarrow \mathbb{R}^{n}$ is order-preserving on $\mathbb{R}_{+}^{n}$ if $g(t, x) \geq g(t, y)$ for any $t \in[0, \infty)$ and any $x, y \in \mathbb{R}_{+}^{n}$ satisfying $x \geq y$.

Definition 3. $f:[0, \infty) \times \mathbb{R}^{n} \rightarrow \mathbb{R}^{n}$ is said to be homogeneous if, for all $t \in[0, \infty)$, all $x \in \mathbb{R}^{n}$, and all real $\lambda>0, f(t, \lambda x)=$ $\lambda f(t, x)$.

It can be similarly shown as in [33] that cooperative vector fields have the following property.

Proposition 4. Let the vector field $f:[0, \infty) \times \mathbb{R}^{n} \rightarrow \mathbb{R}^{n}$ be cooperative. For any two vectors $x, y \in \mathbb{R}_{+}^{n}$, with $x_{i}=y_{i}$ and $x \geq y$, one has $f_{i}(t, x) \geq f_{i}(t, y)$ for $t \in[0, \infty)$.

\section{Main Results}

We first study the reachable set bounding for the continuoustime system (1). Assume that the vector fields $F$ and $G$ satisfy the following assumption.

Assumption A1. (i) There exists a cooperative and homogeneous vector field $f:[0, \infty) \times \mathbb{R}^{n} \rightarrow \mathbb{R}^{n}$ such that $F_{i}(t, x) \operatorname{sign}\left(x_{i}\right) \leq f_{i}(t,|x|)$ for $t \geq 0, x \in \mathbb{R}^{n}, x_{i} \neq 0$, and $i \in\langle n\rangle$.

(ii) There exists a homogeneous and order-preserving vector field $g:[0, \infty) \times \mathbb{R}^{n} \rightarrow \mathbb{R}^{n}$ such that $|G(t, x)| \preceq$ $g(t,|x|)$ for $t \geq 0$ and $x \in \mathbb{R}^{n}$.

Theorem 5. Suppose that Assumption A1 holds, and there exist a vector $v>0$ and a differential function $\mu:\left[-\tau_{\text {max }}, \infty\right) \rightarrow$ $(0, \infty)$ with $\mu(t) \equiv \mu(0)$ for $t \in\left[-\tau_{\max }, 0\right]$, such that $\lim _{t \rightarrow+\infty} \mu(t)=0$,

$$
\begin{aligned}
f(t, v)+g(t, v) & <0, \quad t \geq 0, \\
\frac{f_{i}(t, v)}{v_{i}} \mu(t)+\frac{g_{i}(t, v)}{v_{i}} \mu(t-\tau(t))-\dot{\mu}(t) & <0, \\
t & \geq 0, i \in\langle n\rangle .
\end{aligned}
$$

If there exists a positive constant $\varepsilon_{0}$ satisfying

$$
\frac{-\left|w_{i}(t)\right|}{f_{i}(t, v)+g_{i}(t, v)} \leq \varepsilon_{0}, \quad t \geq 0, \quad i \in\langle n\rangle,
$$


then each solution $x(t)$ of system (1) with the initial condition $x(t)=\phi(t), t \in\left[-\tau_{\text {max }}, 0\right]$, satisfies

$$
\|x(t)\|_{\infty}^{v} \leq \varepsilon_{0}+\widetilde{K} \mu(t), \quad t \geq 0,
$$

where $\widetilde{K}=K / \mu(0), K=\max \left\{0,\|\phi\|-\varepsilon_{0}\right\}$ and $\|\phi\|=$ $\sup _{t \in\left[-\tau_{\max }, 0\right]}\|\phi(t)\|_{\infty}^{v}$.

Proof. Denote

$$
z_{i}(t)=\frac{\left|x_{i}(t)\right|}{v_{i}}-\varepsilon_{0}-\widetilde{K} \mu(t), \quad t \geq 0, \quad i \in\langle n\rangle .
$$

Based on definitions of $\widetilde{K}$ and $\|\phi\|$, we have that $z_{i}(t) \leq 0$ for all $i \in\langle n\rangle$ and $t \in\left[-\tau_{\max }, 0\right]$. Next we will prove that $z_{i}(t) \leq 0$ for all $i \in\langle n\rangle$ and $t \geq 0$. Otherwise, there exists an index $m_{1} \in\langle n\rangle$ and $t_{*}>0$, such that $z_{i}(t) \leq 0$ for $i \in\langle n\rangle$ and $t \in\left[-\tau_{\max }, t_{*}\right], z_{m_{1}}\left(t_{*}\right)=0$, and

$$
\dot{z}_{m_{1}}\left(t_{*}\right) \geq 0
$$

By the definition of $z_{i}(t)$, we have that

$$
\begin{aligned}
\left|x\left(t_{*}\right)\right| & \preceq\left(\varepsilon_{0}+\widetilde{K} \mu\left(t_{*}\right)\right) v, \\
\left|x_{m_{1}}\left(t_{*}\right)\right| & =\left(\varepsilon_{0}+\widetilde{K} \mu\left(t_{*}\right)\right) v_{m_{1}} .
\end{aligned}
$$

Since $f$ is cooperative and homogeneous, we get from Proposition 4 that

$$
\begin{aligned}
f_{m_{1}}\left(t_{*},\left|x\left(t_{*}\right)\right|\right) & \leq f_{m_{1}}\left(t_{*},\left(\varepsilon_{0}+\widetilde{K} \mu\left(t_{*}\right)\right) v\right) \\
& =\left(\varepsilon_{0}+\widetilde{K} \mu\left(t_{*}\right)\right) f_{m_{1}}\left(t_{*}, v\right) .
\end{aligned}
$$

Noting that $t_{*}-\tau\left(t_{*}\right) \in\left[-\tau_{\max }, t_{*}\right]$, we obtain

$$
\left|x\left(t_{*}-\tau\left(t_{*}\right)\right)\right| \preceq\left(\varepsilon_{0}+\widetilde{K} \mu\left(t_{*}-\tau\left(t_{*}\right)\right)\right) v .
$$

Since $g$ is order-preserving and homogeneous, we have

$$
\begin{aligned}
g_{m_{1}} & \left(t_{*},\left|x\left(t_{*}-\tau\left(t_{*}\right)\right)\right|\right) \\
& \leq g_{m_{1}}\left(t_{*},\left(\varepsilon_{0}+\widetilde{K} \mu\left(t_{*}-\tau\left(t_{*}\right)\right)\right) v\right) \\
& =\left(\varepsilon_{0}+\widetilde{K} \mu\left(t_{*}-\tau\left(t_{*}\right)\right)\right) g_{m_{1}}\left(t_{*}, v\right) .
\end{aligned}
$$

Therefore, we can get from Assumption A1, (10), and (12) that

$$
\begin{aligned}
& \dot{z}_{m_{1}}\left(t_{*}\right)=\frac{D_{-}\left|x_{m_{1}}\left(t_{*}\right)\right|}{v_{m_{1}}}-\widetilde{K} \dot{\mu}\left(t_{*}\right) \\
& \leq \frac{1}{v_{m_{1}}}\left[f_{m_{1}}\left(t_{*},\left|x\left(t_{*}\right)\right|\right)\right. \\
& \left.+g_{m_{1}}\left(t_{*},\left|x\left(t_{*}-\tau\left(t_{*}\right)\right)\right|\right)+\left|w_{m_{1}}\left(t_{*}\right)\right|\right] \\
& -\widetilde{K} \dot{\mu}\left(t_{*}\right) \leq \frac{1}{v_{m_{1}}}\left[\varepsilon_{0}\left(f_{m_{1}}\left(t_{*}, v\right)+g_{m_{1}}\left(t_{*}, v\right)\right)\right. \\
& \left.+\left|w_{m_{1}}\left(t_{*}\right)\right|\right]+\widetilde{K}\left[\frac{f_{m_{1}}\left(t_{*}, v\right)}{v_{m_{1}}} \mu\left(t_{*}\right)\right. \\
& \left.+\frac{g_{m_{1}}\left(t_{*}, v\right)}{v_{m_{1}}} \mu\left(t_{*}-\tau\left(t_{*}\right)\right)-\dot{\mu}\left(t_{*}\right)\right] .
\end{aligned}
$$

By using (3) and (5), we have that

$$
\varepsilon_{0}\left(f_{m_{1}}\left(t_{*}, v\right)+g_{m_{1}}\left(t_{*}, v\right)\right)+\left|w_{m_{1}}\left(t_{*}\right)\right| \leq 0
$$

This together with (4) and (13) yields $\dot{z}_{m_{1}}\left(t_{*}\right)<0$, which is a contradiction with (8). As a result, $z_{i}(t) \leq 0$ for $t \geq 0$; that is,

$$
\frac{\left|x_{i}(t)\right|}{v_{i}} \leq \varepsilon_{0}+\widetilde{K} \mu(t), \quad t \geq 0, i \in\langle n\rangle .
$$

It implies that $\|x(t)\|_{\infty}^{v} \leq \varepsilon_{0}+\widetilde{K} \mu(t)$ for $t \geq 0$. This completes the proof of Theorem 5 .

Remark 6. In Theorem 5, conditions (3), (4), and (5) arisen from Assumption Al depend on the time $t$. Such conditions may be less conservative for some cases since they do not require that the disturbance and the time-varying delay are bounded. If we further assume that $f(t, v) \preceq f(v), g(t, v) \preceq$ $g(v)$, and $\left|w_{i}(t)\right| \leq \varepsilon_{i}$ for $t \geq 0$ and $i \in\langle n\rangle$, where $f(v)$ is a time-invariant, cooperative, and homogeneous vector field, $g(v)$ is a time-invariant, homogeneous, and order-preserving vector field, and $\varepsilon_{i}$ are constants, then conditions (3) and (5) are independent of time and hence become verifiable, and $\mu(t)$ can be chosen to be $e^{-\lambda t}$ for some $\lambda>0$ in condition (4).

In the sequel, we study the reachable set bounding for the discrete-time system (2), where the vector fields $F$ and $G$ satisfy the following assumption.

Assumption A2. There exist homogeneous and orderpreserving vector fields $f$ and $g$ such that $|F(k, x)| \preceq f(k,|x|)$ and $|G(k, x)| \preceq g(k,|x|)$ for $k \in \mathbb{N}_{0}$ and $x \in \mathbb{R}^{n}$.

Theorem 7. Assume that Assumption A2 holds, and there exist a vector $v>0$ and a positive sequence $\gamma(k)$ with $\gamma(k) \equiv \gamma(0)$ for $k \in\left\{-d_{\text {max }}, \ldots, 0\right\}$, such that $\lim _{k \rightarrow+\infty} \gamma(k)=0$,

$$
f(k, v)+g(k, v) \prec v, \quad k \in \mathbb{N}_{0},
$$

$$
\begin{aligned}
\frac{f_{i}(k, v)}{v_{i}} \gamma(k)+\frac{g_{i}(k, v)}{v_{i}} \gamma(k-d(k)) & <\gamma(k+1), \\
& k \in \mathbb{N}_{0}, i \in\langle n\rangle .
\end{aligned}
$$

If there exists a positive constant $\varepsilon_{0}$ satisfying

$$
\frac{-\left|w_{i}(k)\right|}{f_{i}(k, v)+g_{i}(k, v)-v_{i}} \leq \varepsilon_{0}, \quad k \in \mathbb{N}_{0}, \quad i \in\langle n\rangle,
$$

then for any sequence $\phi(k) \in \mathbb{R}^{n}$ defined on $\left\{-d_{\text {max }}, \ldots, 0\right\}$, the solution of system (2) satisfies

$$
\|x(k)\|_{\infty}^{v} \leq \varepsilon_{0}+\widehat{K} \gamma(k), \quad k \in \mathbb{N}_{0},
$$

where $\widehat{K}=K / \gamma(0), K=\max \left\{0,\|\phi\|-\varepsilon_{0}\right\}$ and $\|\phi\|=$ $\max _{k \in\left\{-d_{\max }, \ldots, 0\right\}}\|\phi(k)\|_{\infty}^{v}$. 
Proof. We first have

$$
\|x(k)\|_{\infty}^{v} \leq\|\phi\| \leq \varepsilon_{0}+\widehat{K} \gamma(k), \quad k \in\left\{-d_{\max }, \ldots, 0\right\}
$$

Assume that

$$
\|x(k)\|_{\infty}^{v} \leq \varepsilon_{0}+\widehat{K} \gamma(k), \quad k \in\{0,1, \ldots, m\} .
$$

That is,

$$
|x(k)| \preceq\left(\varepsilon_{0}+\widehat{K} \gamma(k)\right) v, \quad k \in\left\{-d_{\max }, \ldots, m\right\} .
$$

Since $f$ and $g$ are homogeneous and order-preserving, we get from Assumption A2 that

$$
\begin{aligned}
& |F(m, x(m))| \preceq f(m,|x(m)|) \\
& \quad \leq\left[\varepsilon_{0}+\widehat{K} \gamma(m)\right] f(m, v), \\
& |G(m, x(m-d(m)))| \preceq g(m,|x(m-d(m))|) \\
& \quad \leq\left[\varepsilon_{0}+\widehat{K} \gamma(m-d(m))\right] g(m, v) .
\end{aligned}
$$

Therefore,

$$
\begin{aligned}
& \frac{\left|x_{i}(m+1)\right|}{v_{i}} \leq \frac{1}{v_{i}}\left[f_{i}(m,|x(m)|)\right. \\
& \left.+g_{i}(m,|x(m-d(m))|)+\left|w_{i}(m)\right|\right] \\
& \quad \leq \frac{1}{v_{i}}\left[\varepsilon_{0}\left(f_{i}(m, v)+g_{i}(m, v)\right)+\left|w_{i}(m)\right|\right] \\
& +\widehat{K}\left[\frac{f_{i}(m, v)}{v_{i}} \gamma(m)+\frac{g_{i}(m, v)}{v_{i}} \gamma(m-d(m))\right],
\end{aligned}
$$

Note that conditions (16) and (18) imply that

$$
\varepsilon_{0}\left(f_{i}(m, v)+g_{i}(m, v)\right)+\left|w_{i}(m)\right| \leq \varepsilon_{0} v_{i}, \quad i \in\langle n\rangle .
$$

This together with (17) and (24) yields that

$$
\frac{\left|x_{i}(m+1)\right|}{v_{i}} \leq \varepsilon_{0}+\widehat{K} \gamma(m+1) \text {. }
$$

By induction, we have that (19) holds. This completes the proof of Theorem 7

Remark 8. In Theorem 7, although conditions (16), (17), and (18) arisen from Assumption A2 depend on the time $t$, they may be less conservative for some cases since they do not require that the disturbance and the time-varying delay are bounded. If we further assume that $f(k, v) \preceq f(v), g(k, v) \preceq$ $g(v)$, and $\left|w_{i}(k)\right| \leq \varepsilon_{i}$ for $k \in \mathbb{N}_{0}$ and $i \in\langle n\rangle$, where $f(v)$ and $g(v)$ are time-invariant, homogeneous, and order-preserving vector fields, and $\varepsilon_{i}$ are constants, then conditions (16) and (18) are independent of time and verifiable, and $\gamma(k)$ can be chosen to be $\eta^{k}$ for some $1>\eta>0$ in condition (17).

\section{Numerical Examples}

We now present two numerical examples to illustrate the main results of this paper.

Example 1. Consider the continuous-time nonlinear timevarying system given by (1) with

$$
\begin{aligned}
F(t, x)= & {\left[\begin{array}{cc}
-5+\sin t & \frac{t}{1+t} \\
\cos t & -5-\sin t
\end{array}\right]\left[\begin{array}{l}
x_{1} \\
x_{2}
\end{array}\right] } \\
& +\sqrt{x_{1}^{2}+x_{2}^{2}}\left[\begin{array}{c}
\sin t \\
\frac{t}{1+t}
\end{array}\right], \\
G(t, x)= & \frac{x_{1} x_{2}}{\sqrt{x_{1}^{2}+x_{2}^{2}}}\left[\begin{array}{l}
\cos t \\
\sin t
\end{array}\right], \\
w(t)= & 0.05\left[\begin{array}{l}
\sin t \\
\cos t
\end{array}\right], \\
\tau(t)= & 2+\sin t .
\end{aligned}
$$

It can be seen that $F$ and $G$ satisfy Assumption A1 with

$$
\begin{aligned}
& f(t, x) \equiv\left[\begin{array}{c}
-4 x_{1}+x_{2}+\sqrt{x_{1}^{2}+x_{2}^{2}} \\
x_{1}-4 x_{2}+\sqrt{x_{1}^{2}+x_{2}^{2}}
\end{array}\right], \\
& g(t, x) \equiv \frac{x_{1} x_{2}}{\sqrt{x_{1}^{2}+x_{2}^{2}}}\left[\begin{array}{l}
1 \\
1
\end{array}\right],
\end{aligned}
$$

$$
t \geq 0 \text {. }
$$

Condition (3) reduces to

$$
\begin{gathered}
-4 v_{1}+v_{2}+\sqrt{v_{1}^{2}+v_{2}^{2}}+\frac{v_{1} v_{2}}{\sqrt{v_{1}^{2}+v_{2}^{2}}}<0 \\
v_{1}-4 v_{2}+\sqrt{v_{1}^{2}+v_{2}^{2}}+\frac{v_{1} v_{2}}{\sqrt{v_{1}^{2}+v_{2}^{2}}}<0
\end{gathered}
$$

Let $\mu(t)=e^{-\lambda t}$ for $\lambda>0$. Then, condition (4) holds if

$$
\begin{gathered}
\frac{-4 v_{1}+v_{2}+\sqrt{v_{1}^{2}+v_{2}^{2}}}{v_{1}}+\frac{v_{1} v_{2}}{v_{1} \sqrt{v_{1}^{2}+v_{2}^{2}}} e^{3 \lambda}+\lambda<0, \\
\frac{v_{1}-4 v_{2}+\sqrt{v_{1}^{2}+v_{2}^{2}}}{v_{2}}+\frac{v_{1} v_{2}}{v_{2} \sqrt{v_{1}^{2}+v_{2}^{2}}} e^{3 \lambda}+\lambda<0 .
\end{gathered}
$$




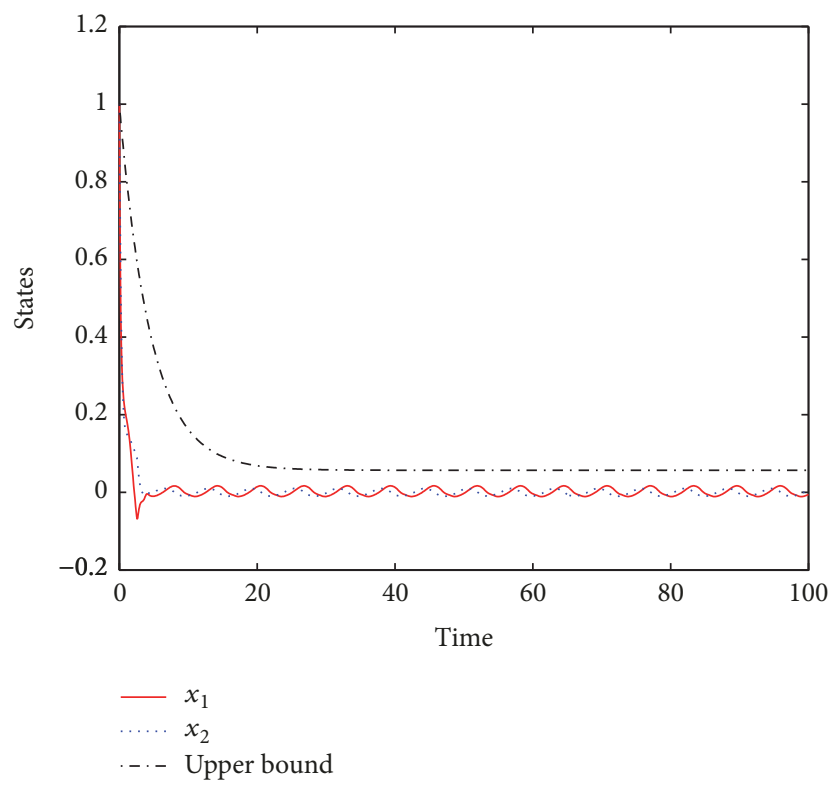

FIGURE 1: State and its upper bound of continuous-time system (1).

For given $v$ and $\lambda$ satisfying (29) and (30), condition (5) yields that

$$
\begin{gathered}
\varepsilon_{0}=\min \left\{\frac{0.05}{4 v_{1}-v_{2}-\sqrt{v_{1}^{2}+v_{2}^{2}}-2 v_{1} v_{2} / \sqrt{v_{1}^{2}+v_{2}^{2}}},\right. \\
\left.\frac{0.05}{-v_{1}+4 v_{2}-\sqrt{v_{1}^{2}+v_{2}^{2}}-2 v_{1} v_{2} / \sqrt{v_{1}^{2}+v_{2}^{2}}}\right\} .
\end{gathered}
$$

Set $v=(1,1)^{T}$ and $\lambda=0.219$. A straightforward computation yields that conditions (29) and (30) hold, and $\varepsilon_{0}=0.057$. If we choose the initial condition $\phi(t)=(1,1)^{T}$ for $t \in[-3,0]$, then $\|\phi\|=1$ and $\widetilde{K}=0.943$. By using Theorem 5 , the solution $x(t)$ of this system satisfies $\|x(t)\|_{\infty}^{v} \leq 0.057+0.943 e^{-0.219 t}$ for all $t \geq 0$. The simulation result is presented in Figure 1 .

Generally speaking, the minimal parameter constant $\varepsilon_{0}$ can be determined by the following nonlinear programming problem: minimize $\varepsilon_{0}$ defined by (31) subject to $v>0, \lambda>0$, and (29) and (30).

Example 2. Consider the discrete-time nonlinear timevarying system given by (2) with

$$
\begin{aligned}
& F(k, x)=0.2 \sqrt{x_{1}^{2}+x_{2}^{2}}\left[\begin{array}{c}
\sin k \\
\frac{k}{1+k}
\end{array}\right], \\
& G(k, x)=\frac{x_{1} x_{2}}{2}\left[\begin{array}{c}
\frac{\cos k}{\sqrt{x_{1}^{2}+x_{2}^{2}}} \\
\frac{\sin k}{\sqrt{2 x_{1}^{2}+3 x_{2}^{2}}}
\end{array}\right],
\end{aligned}
$$

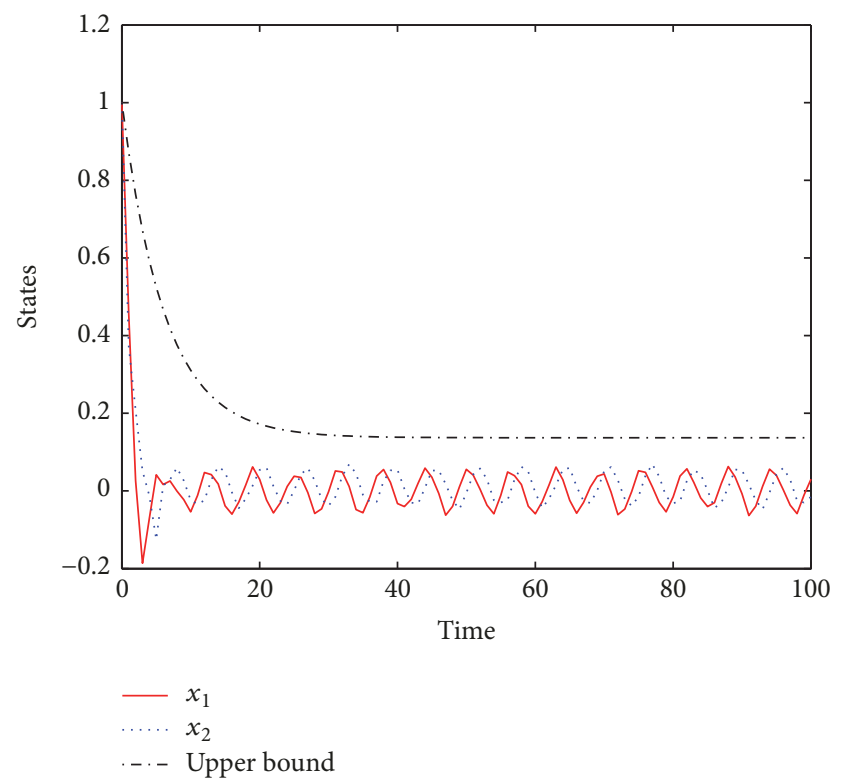

FIGURE 2: State and its upper bound of discrete-time system (2).

$$
\begin{aligned}
& w(k)=0.05\left[\begin{array}{c}
\cos k \\
\sin k
\end{array}\right], \\
& d(k)=2+\sin (0.5 k \pi) .
\end{aligned}
$$

It is easy to see that $F$ and $G$ satisfy Assumption A2 with

$$
\begin{aligned}
& f(k, x) \equiv 0.2 \sqrt{x_{1}^{2}+x_{2}^{2}}\left[\begin{array}{l}
1 \\
1
\end{array}\right], \\
& g(k, x) \equiv \frac{x_{1} x_{2}}{2}\left[\begin{array}{c}
\frac{1}{\sqrt{x_{1}^{2}+x_{2}^{2}}} \\
\frac{1}{\sqrt{2 x_{1}^{2}+3 x_{2}^{2}}}
\end{array}\right]
\end{aligned}
$$

By choosing the vector $v=(1,1)^{T}$ and $\gamma(k)=\eta^{k}$ with $\eta=0.852$, we have that both (16) and (17) hold. A further computation yields that $\varepsilon_{0}=0.137$. By choosing the initial condition $\phi(k)=(1,1)^{T}$, we have $\|\phi\|=1$ and $\widehat{K}=0.863$. By using Theorem 7, the solution $x(k)$ of the system satisfies $\|x(k)\|_{\infty}^{\nu} \leq 0.137+0.863(0.852)^{k}$ for all $k \in N_{0}$. The simulation result is presented in Figure 2.

\section{Conclusion}

In this paper, the problem of reachable set bounding for a class of continuous-time and discrete-time nonlinear timevarying systems with delay has been investigated, where the involved disturbance input and time-varying delay may be unbounded. By using an approach which is different from the Lyapnov-Krasovskii functional method, we establish sufficient conditions such that all the state trajectories 
of the system converge asymptotically within a ball. Two illustrative examples and simulation results are given to show the effectiveness of the obtained results.

\section{Conflicts of Interest}

The authors declare no conflicts of interest.

\section{Acknowledgments}

This paper is supported by the National Natural Science Foundation of China under Grant nos. 61473133, 11671227, and 61374074 and the Natural Science Foundation of Shandong Province under Grant no. JQ201119.

\section{References}

[1] J. Lygeros, C. Tomlin, and S. Sastry, "Controllers for reachability specifications for hybrid systems," Automatica, vol. 35, no. 3, pp. 349-370, 1999.

[2] J. Lam, B. Zhang, Y. Chen, and S. Xu, "Reachable set estimation for discrete-time linear systems with time delays," International Journal of Robust and Nonlinear Control, vol. 25, no. 2, pp. 269281, 2015.

[3] C. Shen and S. Zhong, "The ellipsoidal bound of reachable sets for linear neutral systems with disturbances," Journal of The Franklin Institute, vol. 348, no. 9, pp. 2570-2585, 2011.

[4] Z. Zuo, Z. Wang, Y. Chen, and Y. Wang, "A non-ellipsoidal reachable set estimation for uncertain neural networks with time-varying delay," Communications in Nonlinear Science and Numerical Simulation, vol. 19, no. 4, pp. 1097-1106, 2014.

[5] B. Zhang, J. Lam, and S. Xu, "Reachable set estimation and controller design for distributed delay systems with bounded disturbances," Journal of The Franklin Institute, vol. 351, no. 6, pp. 3068-3088, 2014.

[6] Z. Zuo, D. W. Ho, and Y. Wang, "Reachable set estimation for linear systems in the presence of both discrete and distributed delays," IET Control Theory \& Applications, vol. 5, no. 15, pp. 1808-1812, 2011.

[7] Z. Zuo, Y. Fu, and Y. Wang, "Results on reachable set estimation for linear systems with both discrete and distributed delays," IET Control Theory \& Applications, vol. 6, no. 14, pp. 2346-2350, 2012.

[8] Y. Sun and J. Qi, "Note on exponential stability of certain nonlinear differential systems with time-varying delays," Applied Mathematics Letters, vol. 25, no. 12, pp. 2240-2245, 2012.

[9] Y. Li, Y. Sun, and F. Meng, "New criteria for exponential stability of switched time-varying systems with delays and nonlinear disturbances," Nonlinear Analysis: Hybrid Systems, vol. 26, pp. 284-291, 2017.

[10] Y. Li, Y. Sun, F. Meng, and Y. Tian, "Exponential stabilization of switched time-varying systems with delays and disturbances," Applied Mathematics and Computation, vol. 324, pp. 131-140, 2018.

[11] G. Zong, H. Ren, and L. Hou, "Finite-time stability of interconnected impulsive switched systems," IET Control Theory \& Applications, vol. 10, no. 6, pp. 648-654, 2016.

[12] Y. Yin, G. Zong, and X. Zhao, "Improved stability criteria for switched positive linear systems with average dwell time switching," Journal of The Franklin Institute, vol. 354, no. 8, pp. 3472-3484, 2017.
[13] Y. Sun, Y. Tian, and X.-J. Xie, "Stabilization of positive switched linear systems and its application in consensus of multiagent systems," Institute of Electrical and Electronics Engineers Transactions on Automatic Control, vol. 62, no. 12, pp. 6608-6613, 2017.

[14] Y. Sun, Z. Wu, and F. Meng, "Common weak linear copositive Lyapunov functions for positive switched linear systems," Complexity, vol. 2018, 7 pages, 2018.

[15] F. Meng and Y. Huang, "Interval oscillation criteria for a forced second-order nonlinear differential equations with damping," Applied Mathematics and Computation, vol. 218, no. 5, pp. 18571861, 2011.

[16] H. Liu, F. Meng, and P. Liu, "Oscillation and asymptotic analysis on a new generalized Emden-Fowler equation," Applied Mathematics and Computation, vol. 219, no. 5, pp. 2739-2748, 2012.

[17] J. Shao, Z. Zheng, and F. Meng, "Oscillation criteria for fractional differential equations with mixed nonlinearities," Advances in Difference Equations, p. 323, 2013.

[18] J. Shao, F. Meng, and Z. Zheng, "Oscillation theorems for linear matrix Hamiltonian systems," Applied Mathematics and Computation, vol. 253, pp. 402-409, 2015.

[19] H. Liu and F. Meng, "Interval oscillation criteria for secondorder nonlinear forced differential equations involving variable exponent," Advances in Difference Equations, Paper No. 291, 14 pages, 2016.

[20] E. Fridman and U. Shaked, "On reachable sets for linear systems with delay and bounded peak inputs," Automatica, vol. 39, no. 11, pp. 2005-2010, 2003.

[21] J.-H. Kim, "Improved ellipsoidal bound of reachable sets for time-delayed linear systems with disturbances," Automatica, vol. 44, no. 11, pp. 2940-2943, 2008.

[22] Z. Zuo, Y. Chen, Y. Wang, D. W. Ho, M. Z. Chen, and H. Li, "A note on reachable set bounding for delayed systems with polytopic uncertainties," Journal of The Franklin Institute, vol. 350, no. 7, pp. 1827-1835, 2013.

[23] Z. Zuo, D. W. Ho, and Y. Wang, "Reachable set bounding for delayed systems with polytopic uncertainties: the maximal Lyapunov-Krasovskii functional approach," Automatica, vol. 46, no. 5, pp. 949-952, 2010.

[24] P. T. Nam and P. N. Pathirana, "Further result on reachable set bounding for linear uncertain polytopic systems with interval time-varying delays," Automatica, vol. 47, no. 8, pp. 1838-1841, 2011.

[25] N. D. That, P. T. Nam, and Q. P. Ha, "Reachable set bounding for linear discrete-time systems with delays and bounded disturbances," Journal of Optimization Theory and Applications, vol. 157, no. 1, pp. 96-107, 2013.

[26] L. V. Hien and H. M. Trinh, "A new approach to state bounding for linear time-varying systems with delay and bounded disturbances," Automatica, vol. 50, no. 6, pp. 1735-1738, 2014.

[27] N. Zhang, Y. Sun, and P. Zhao, "State bounding for homogeneous positive systems of degree one with time-varying delay and exogenous input," Journal of The Franklin Institute, vol. 354, no. 7, pp. 2893-2904, 2017.

[28] P. T. Nam, P. N. Pathirana, and H. Trinh, "Reachable set bounding for nonlinear perturbed time-delay systems: the smallest bound," Applied Mathematics Letters, vol. 43, pp. 68-71, 2015.

[29] J. Qi and Y. Sun, "Global exponential stability of certain switched systems with time-varying delays," Applied Mathematics Letters, vol. 26, no. 7, pp. 760-765, 2013. 
[30] H. R. Feyzmahdavian, T. Charalambous, and M. Johansson, "Exponential stability of homogeneous positive systems of degree one with time-varying delays," Institute of Electrical and Electronics Engineers Transactions on Automatic Control, vol. 59, no. 6, pp. 1594-1599, 2014.

[31] Y. Sun and F. Meng, "Reachable set estimation for a class of nonlinear time-varying systems," Complexity, vol. 2017, Article ID 5876371, 6 pages, 2017.

[32] J. K. Hale and S. M. Verduyn Lunel, Introduction to FunctionalDifferential Equations, Springer, Berlin, Germany, 1993.

[33] H. L. Smith, Monotone Dynamical Systems: An Introduction to the Theory of Competitive and Cooperative Systems, American Mathematical Society, Raleigh, NC, USA, 1995. 


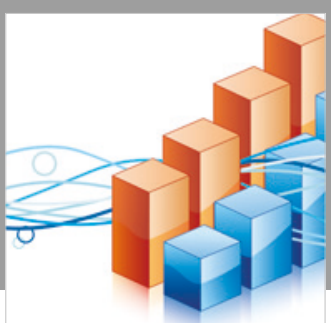

Advances in

Operations Research

\section{-n-m}
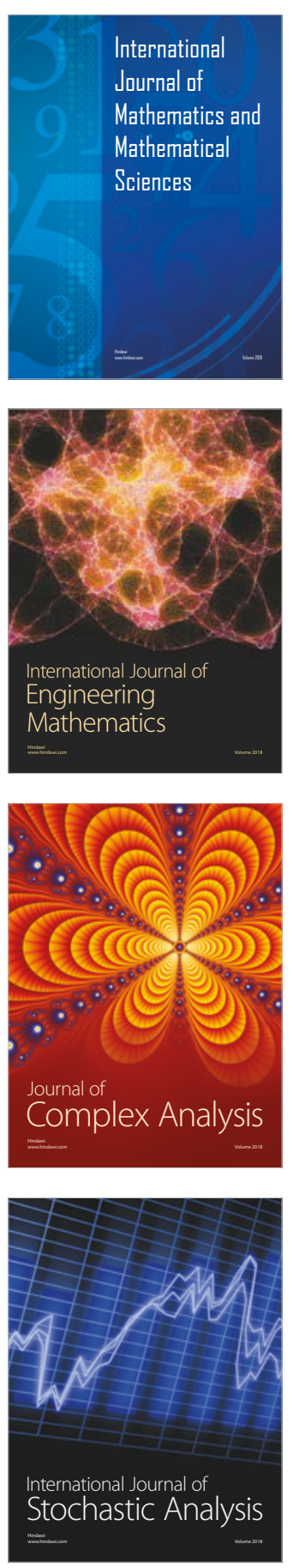
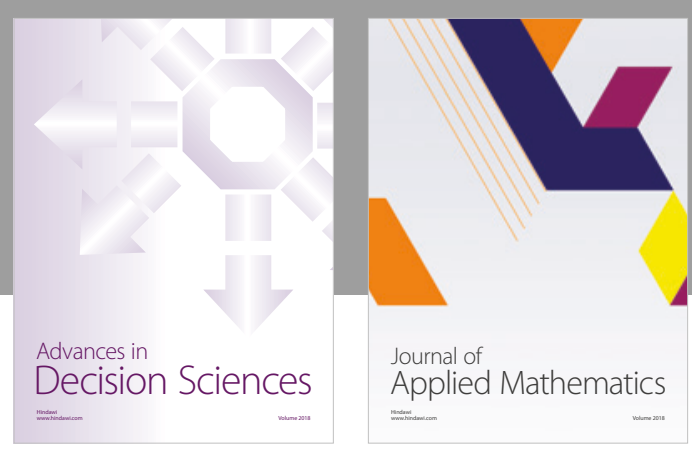

Journal of

Applied Mathematics
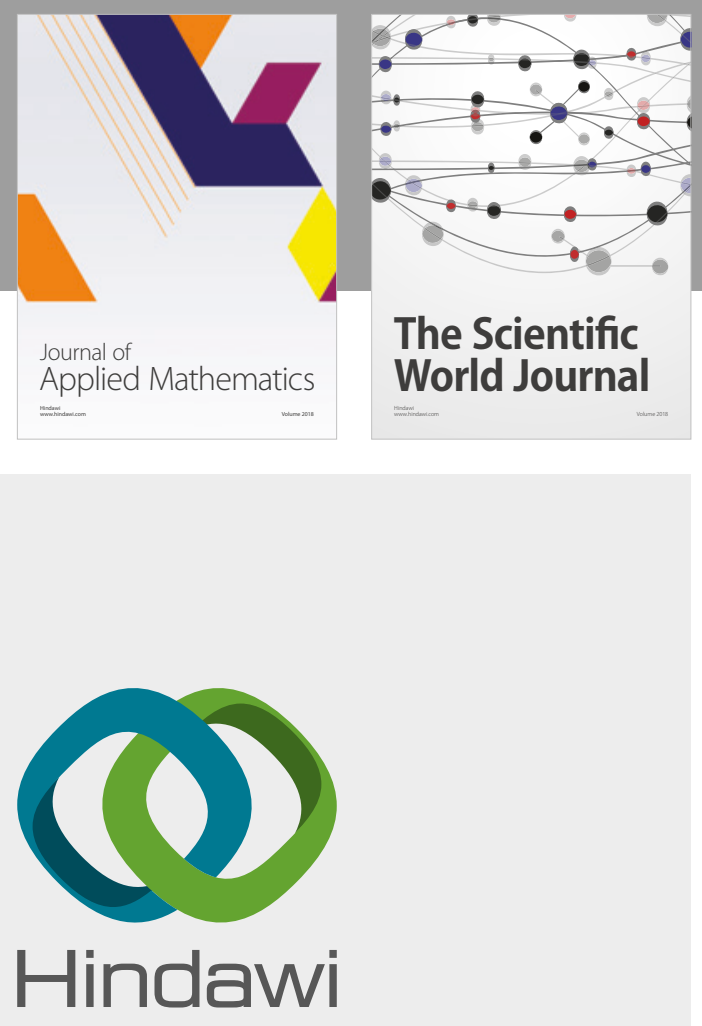

Submit your manuscripts at

www.hindawi.com

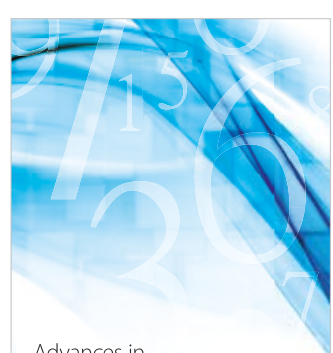

Advances in
Numerical Analysis
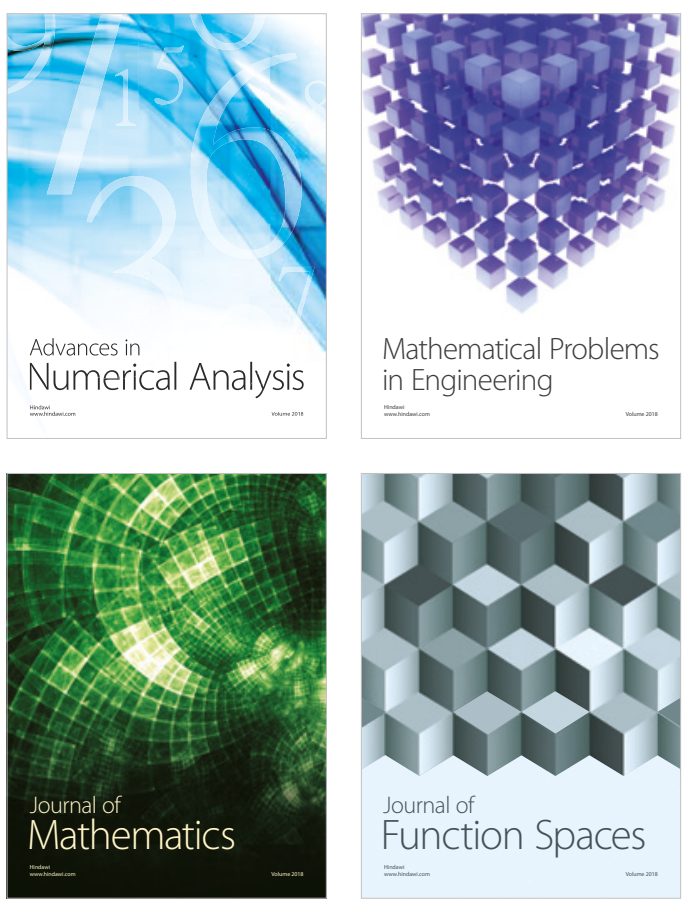

Mathematical Problems in Engineering

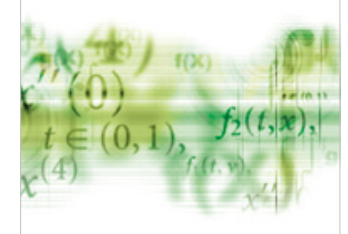

International Journal of

Differential Equations

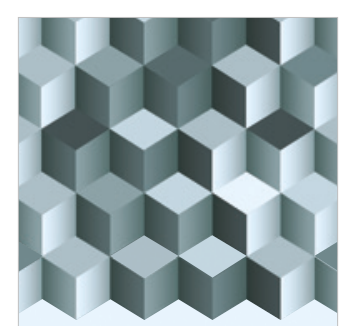

Journal of

Function Spaces

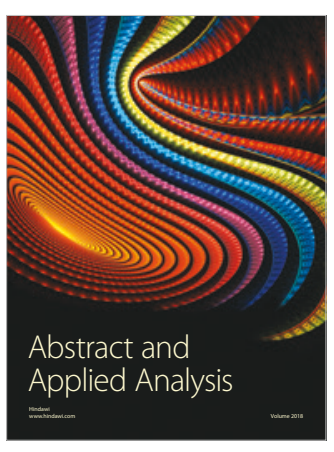

The Scientific

World Journal

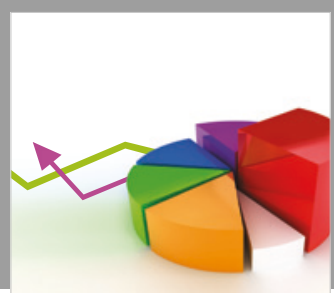

Journal of

Probability and Statistics
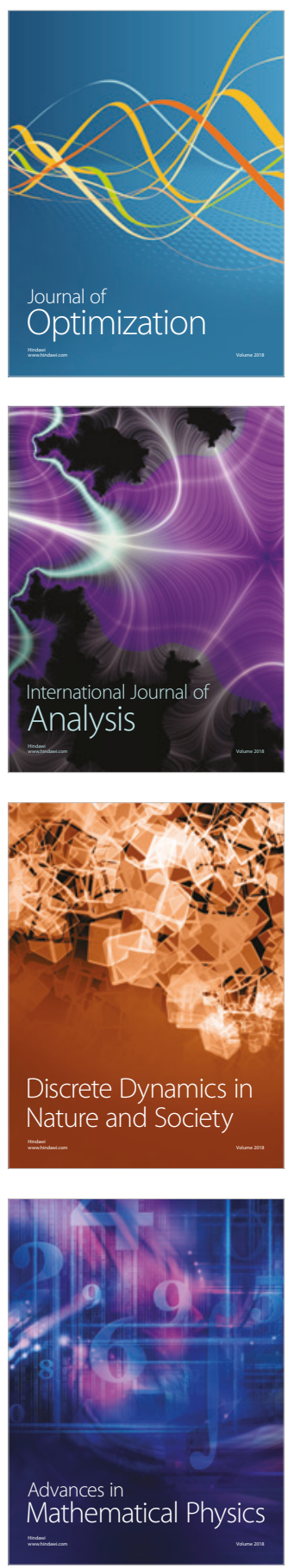